SMASIS2012-8255

\title{
INFLUENCE OF SENSOR STATISTICS ON PIEZOELECTRIC AND MAGNETO- ELASTIC DAMAGE DETECTION
}

\author{
David Conrad, Andrei Zagrai, Daniel Meisner \\ Department of Mechanical Engineering, New Mexico Institute of Mining and Technology \\ 801 Leroy Place, Socorro, NM, 87801, USA
}

\section{ABSTRACT}

Increasing complexity of aerospace structures facilitates a growing need for structural health monitoring (SHM) systems capable of real-time active damage detection. A variety of sensing approaches have been demonstrated using embedded ultrasonic sensors such as piezoelectric wafer active sensors (PWAS) and magneto-elastic active sensors (MEAS). Common methodologies consider wave propagation (pitchcatch or pulse-echo) and standing wave (vibration or impedance) techniques with damage detection capabilities dependent upon structural geometry, material characteristics, distance to damage and damage size/orientation. While recent studies have employed damage detection and classification approaches that are dependent on cumulative statistics, this study explores the contribution of sensor parameters and experimental setup variability on the damage detection scheme. The impact of variability in PWAS and MEAS are considered on sensor use in ultrasonic and magneto-mechanical impedance damage detection.

In order to isolate sensor parameters, measurements were conducted with PWAS in free-free boundary conditions. Variability of PWAS parameters was evaluated by measuring the sensors impedance response. An analytical model of PWAS was used to estimate sensor parameters and to determine their variability. Additionally, experiments using MEAS were performed that demonstrate variation of magneto-mechanical impedance during structural dynamic tests. From these experiments the importance of sensor setup is discussed and its contribution into the overall detection scheme is explored.

\section{INTRODUCTION}

Structural health monitoring can be a useful tool for inferring information about structural integrity. The early identification of structural damage is critical in many cases for maintaining a safe and functional operating environment. However, a large number of factors can influence an SHM systems ability to accurately determine the integrity of the structure. Traditionally, the evaluation of an SHM system involves considering a specific technology with a fixed sensor type and damage detection methodology. These evaluations then study the influence of contributing factors within the scope of the specific SHM system. The statistical information collected for these situations reflect a cumulative contribution of all SHM factors, such as sensors, electronics, bonds, structure, environment, etc. The SHM system will need to be reevaluated if any factor is changed. An alternative approach is suggested in which contributions of individual SHM components into the cumulative statistics are isolated.

\section{PIEZOELECTRIC SENSOR CHARACTERISTICS}

The performance of the overall SHM system is substantially influenced by the choice of sensors. In order to understand the contribution of the sensor to the entire SHM system, it is important to understand the influence of the different factors on the sensors performance. Sensor characteristics may have a different influence on the sensors performance in transmission versus reception. Sensors are evaluated in static and dynamic conditions. Static characteristics include sensor sensitivity, linearity, repeatability and etc. Dynamic characteristics can be divided into steady state and transient. In the following study we mostly focus on the electro-mechanical impedance response, which is used to characterize piezoelectric sensors in steady state dynamic condition.

Piezoelectric wafer active sensors are commonly used in SHM and present an excellent specimen for this study. They are rather well understood and are characterized by their electro-mechanical (E/M) impedance defined as the frequency dependent ratio of voltage to current [1]. Therefore, in order to explore the effects of parameters variability on the performance of the sensor, it is necessary to evaluate variability of $\mathrm{E} / \mathrm{M}$ impedance under similar conditions.

\section{PWAS Impedance Statistics}

PWAS is coupled to the host structure with a thin layer of adhesive bond. In order to decouple the effect of bond layer and medium on the PWAS statistics, impedance measurements were performed on PWAS in a free-free boundary condition without soldered leads. A sample size of 30 was selected so that population estimates may be performed 
using statistical processing for large sample sets. The sample batch consisted of 30 APC-851 circular PWAS from American Piezo Ceramics, Inc. The sensors have a $7 \mathrm{~mm}$ diameter and a thickness of $0.25 \mathrm{~mm}$. The batch of PWAS was manufactured with a wrap around electrode as shown in Figure 1. The wrap around electrode is beneficial for many embedded sensor applications; however, it increases the complexity of electromechanical transformation within the sensor and respective analytical and numerical descriptions.

(a)

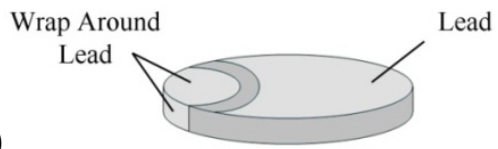

(b)

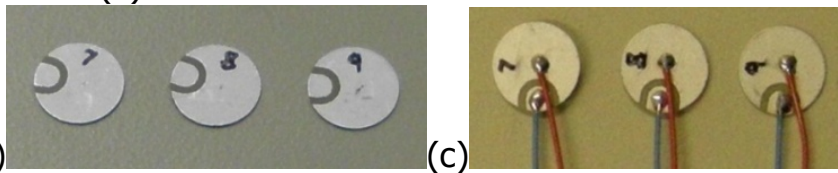

Figure 1 Circular PWAS diagram with wrap around electrode. c) Test samples for free-free test fixture. d) Test samples with soldered leads.

Two sets of impedance and admittance measurements were taken. First, the 30 samples were tested using a fixture to simulate a free-free boundary condition of the PWAS without soldered leads, see Figure $2 \mathrm{a}$. The fixture consisted of a pair of thin electrical probes mounted onto a C-clamp. The fixture clamps a sensor between the two probes which are connected to the impedance analyzer. This allows the probes to be positioned at the center of the PWAS and function as measurement terminals. The free-free fixture was placed on a foam block during measurements. All 30 sensors were oriented with the top electrode making electrical contact with the fixtures terminal that was connected to the positive voltage output of the impedance analyzer. The C-clamp's t-handle was turned to approximately the same angle for each measurement to ensure similar clamping forces for all 30 measurements. The second measurement set was taken after leads were soldered to the electrodes. For this measurement, the PWAS were suspended by their leads so the sensors were not in contact with any surface. The wrap around lead was connected to ground for all 30 samples.

An initial E/M impedance measurement was taken with one sensor in the free-free test fixture using a broad frequency range. Three $\mathrm{E} / \mathrm{M}$ impedance measurement ranges were chosen based on the $\mathrm{E} / \mathrm{M}$ resonance locations recorded in this initial sweep. The first range chosen was from $200-1000$ $\mathrm{kHz}$ with a resolution of $500 \mathrm{~Hz}$. The second range was from $250-400 \mathrm{kHz}$ with a $100 \mathrm{~Hz}$ resolution. The third measurement range was $8-11 \mathrm{MHz}$ with a $4 \mathrm{kHz}$ resolution. The third measurement range captures the fundamental resonance of the thickness mode, while the other two ranges capture an in-plane resonance. Both sets of measurements were taken using an HP4192A impedance analyzer seen in Figure $2 b$. (a)

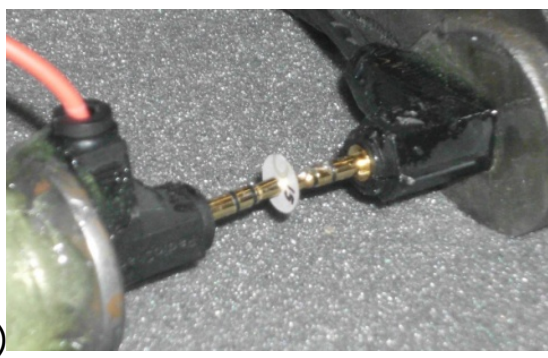

(b)

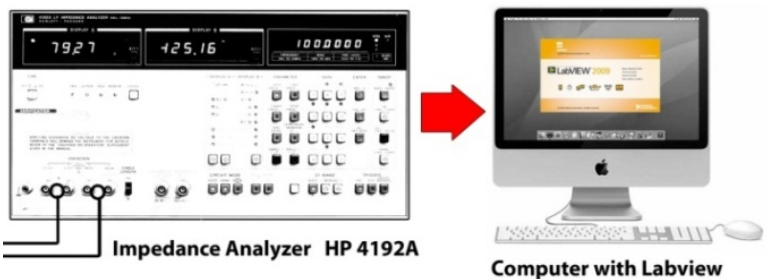

Figure 2 a) Free-free test fixture b) HP4192A setup.

After collecting test data for free-free PWAS conditions depicted in Figure 2a, leads were soldered for all participating sensors and impedance data were taken again. Impedance tests yielded collections of data for all

The data was processed to determine the frequencies of the primary resonance and anti-resonance. The zero-crossing frequency for the admittance phase is taken as a measure of the undamped resonance frequency. Likewise, the zero-crossing frequency for the impedance phase is taken as a measure of the undamped anti-resonance frequency. The zero-crossing frequency was calculated by performing a linear interpolation between points lying above and below the zero crossing. Figure 3 shows an example of the admittance phase zero-crossing measurements.

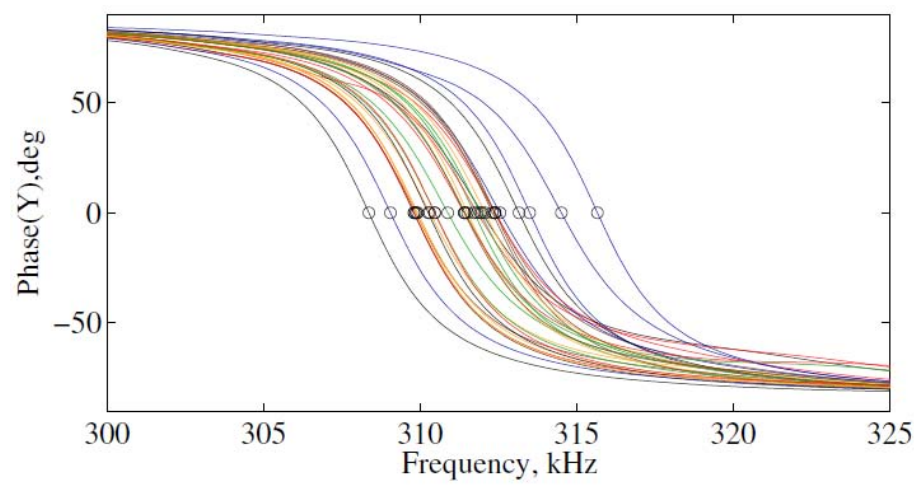

Figure 3 Admittance phase and resonance frequencies determined as zero crossings.

Figure 4 and Figure 5 show representative impedance and admittance curves collected for PWAS in the fixture and with soldered leads. 

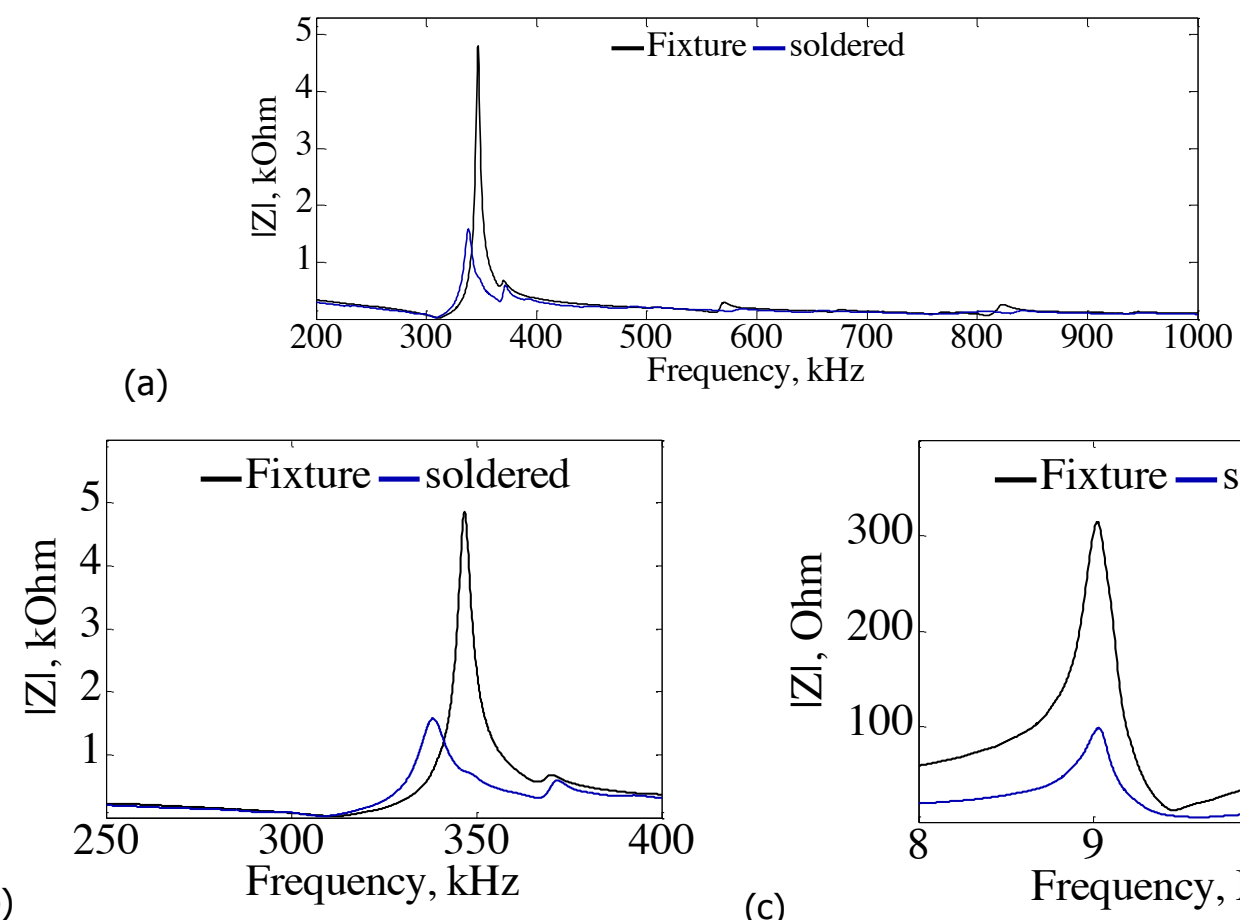

(c)

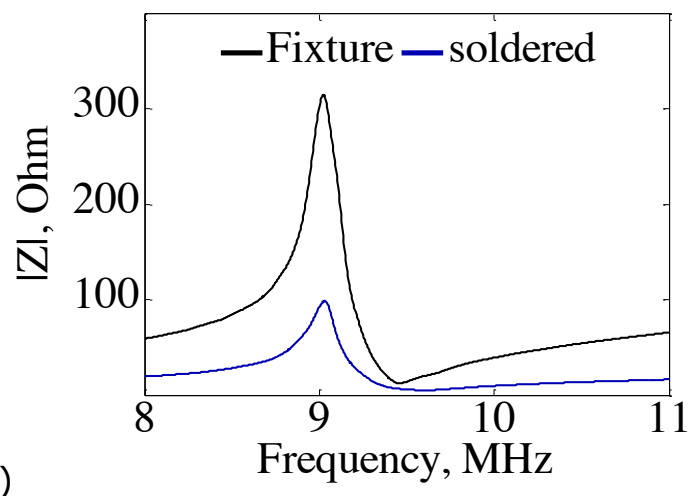

Figure 4 Test data from PWAS \#4 comparing the impedance magnitude measurements using the freefree fixture to the measured with soldered leads. a) shows $200-1000 \mathrm{kHz}$ range, b) shows $250-400 \mathrm{kHz}$ range, and c) shows $8-11 \mathrm{MHz}$ range.

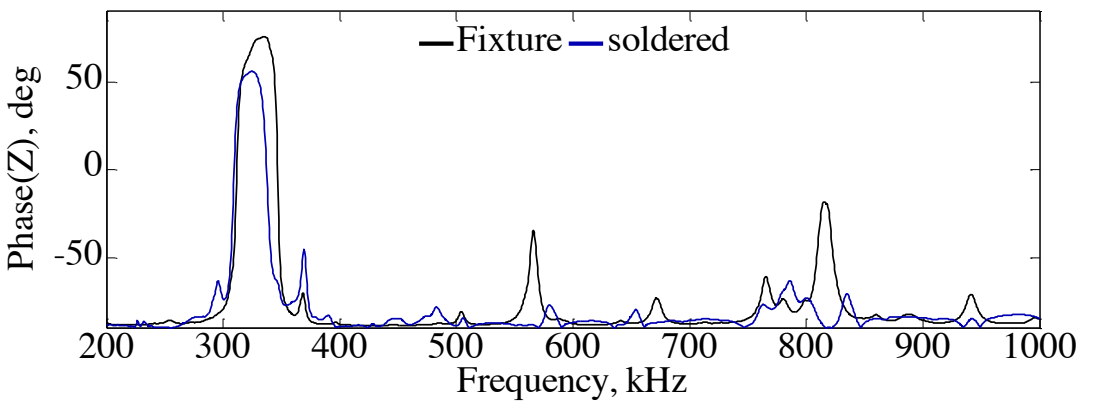

(a)
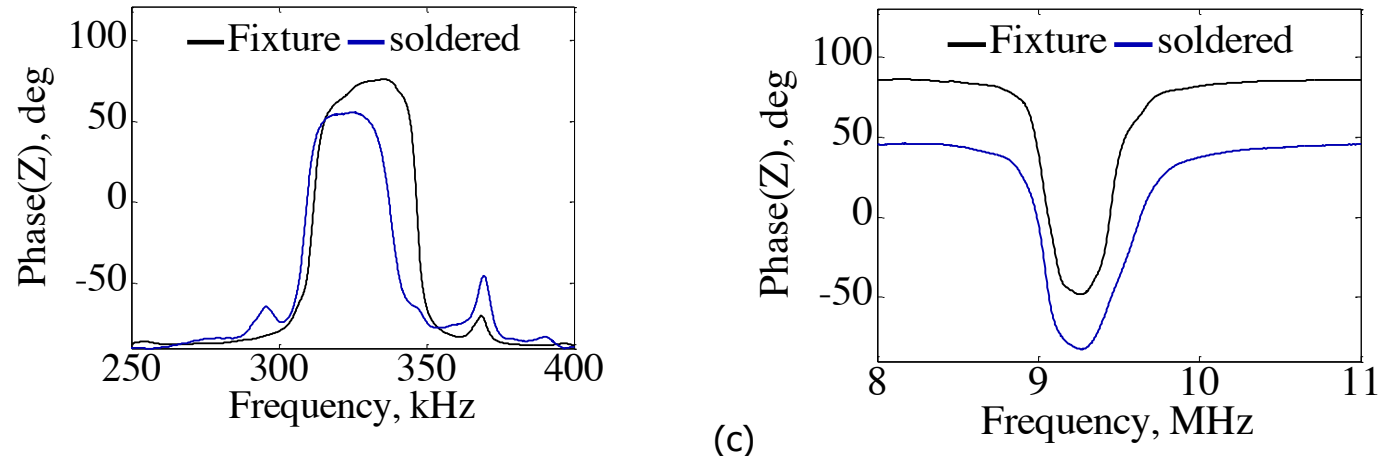

Figure 5 Test data from PWAS \#4 comparing the impedance phase measurements using the free-free fixture to the measured with soldered leads. a) shows $200-1000 \mathrm{kHz}$ range, b) shows $250-$ $400 \mathrm{kHz}$ range, and c) shows $8-11 \mathrm{MHz}$ range. 


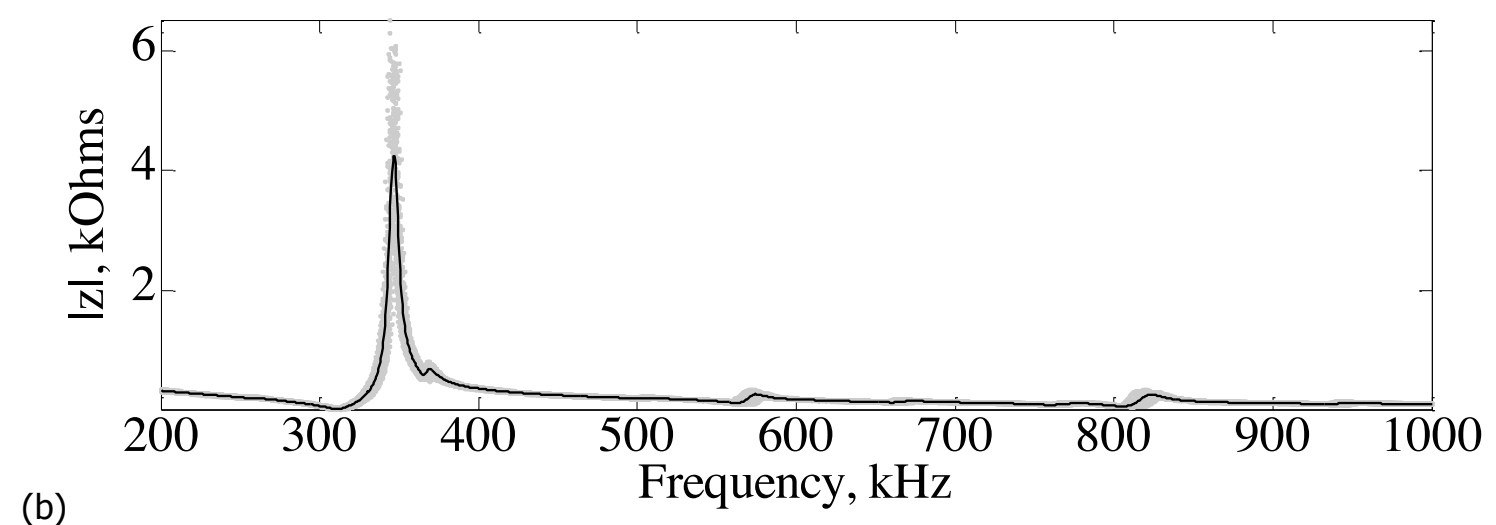

(b)

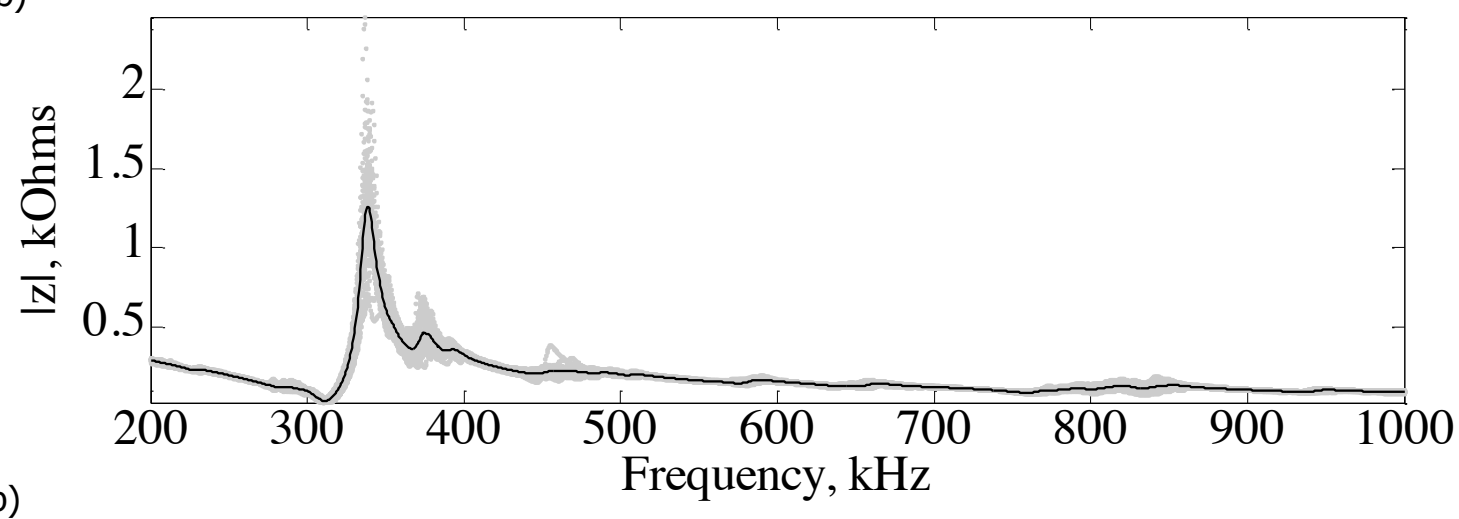

Figure 6 Electro-mechanical impedance response of (a) free PWAS measured in fixture and (b) free PWAS with soldered leads. Mean response is indicated as solid line.

To quantify the PWAS measurement results, a sample mean and standard deviation were calculated for the in-plane and thickness undamped resonances and anti-resonances using equation below [2].

$$
\bar{x}=\frac{\Sigma x}{n}=\frac{x_{1}+x_{2}+x_{3}+\cdots+x_{n}}{n}
$$

Similarly the formula for calculating a samples variance was used:

$$
s=\sqrt{\frac{\sum(x-\bar{x})^{2}}{n-1}}
$$

Results of the calculations are summarized in Table 1 showing the sample mean and standard deviations for the inplane resonance and anti-resonance. It should be noted that the standard deviation for the resonance and anti-resonance is higher for the soldered case. It is understandable that the soldering process creates more variability in the impedance and admittance response of the sensors as illustrated in Figure 6. It is also important to note that the anti-resonance frequency decreases $8.7 \mathrm{kHz}$ for the soldered case while the resonance frequency increases $0.9 \mathrm{kHz}$ for the soldered case.
Table 1 Sample mean and standard deviations for inplane resonances

\begin{tabular}{|c|c|c|}
\hline Sample Case & $\begin{array}{c}\text { Sample Mean } \\
(\mathrm{kHz})\end{array}$ & $\begin{array}{c}\text { Sample } \\
\text { Standard } \\
\text { Deviation }(\mathrm{kHz})\end{array}$ \\
\hline In Plane Resonance (Fixture) & 311.4 & 1.58 \\
\hline In Plane Resonance (Soldered) & 312.3 & 1.65 \\
\hline $\begin{array}{c}\text { In Plane Anti-resonance } \\
\text { (Fixture) }\end{array}$ & 346.5 & 1.61 \\
\hline $\begin{array}{c}\text { In Plane Anti-resonance } \\
\text { (Soldered) }\end{array}$ & 337.8 & 1.82 \\
\hline
\end{tabular}

A theoretical Gaussian distribution for each sample variable was calculated using the sample's mean and standard deviation. The theoretical distributions were scaled and plotted on top of the histograms as seen in Figure 7. The histograms appear to be in reasonable agreement with the Gaussian distribution for the in-plane resonance and anti-resonance. Plots of sample residuals were generated to assess any bias errors that may be present in the data. No unusual trends were observed and the residuals appear normally distributed. 


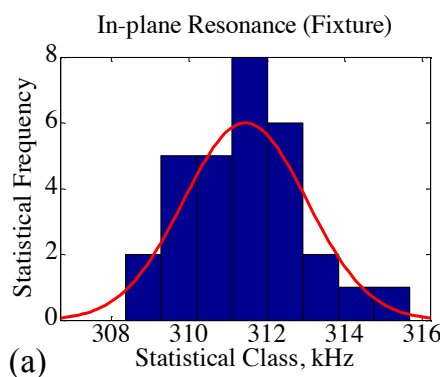

(a)

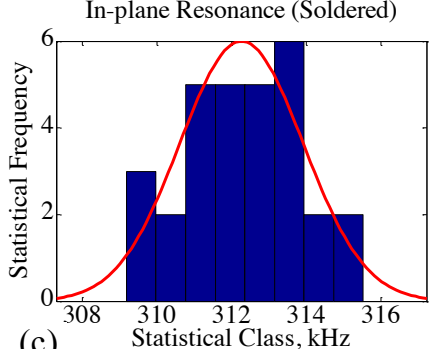

(c)

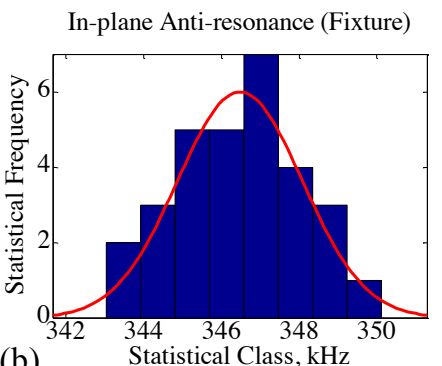

(b) In-plane Anti-resonance (Soldered)

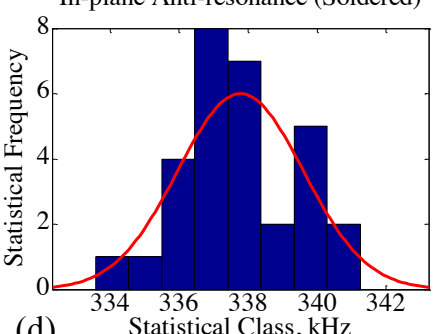

(d)

In addition to calculating statistical parameters for the resonance and anti-resonance frequencies, statistical parameters were also calculated for the amplitudes of the resonance and anti-resonance peaks. Table 2 and Table 3 with respective Figure 9 and Figure 10 show the calculated statistics for the inplane and thickness resonance and anti-resonance peaks. The amplitude of those peaks clearly decreases except for the thickness anti-resonance which increases. In all cases the standard deviation increases as a percentage of mean for the soldered samples.

Table 2 Sample mean and standard deviations for inplane resonance amplitudes.

\begin{tabular}{|l|c|c|}
\hline \multicolumn{1}{|c|}{ Sample Case } & Sample Mean & $\begin{array}{c}\text { Sample } \\
\text { Standard } \\
\text { Deviation }\end{array}$ \\
\hline In Plane Resonance (Fixture) & $61.9 \mathrm{mS}$ & $5.5 \mathrm{mS}$ \\
\hline In Plane Resonance (Soldered) & $36.7 \mathrm{mS}$ & $5.0 \mathrm{mS}$ \\
\hline In Plane Anti-resonance (Fixture) & $5602 \Omega$ & $530 \Omega$ \\
\hline In Plane Anti-resonance (Soldered) & $1403 \Omega$ & $339 \Omega$ \\
\hline
\end{tabular}

Table 3 Sample mean and standard deviations for thickness resonance amplitudes.

\begin{tabular}{|l|c|c|}
\hline \multicolumn{1}{|c|}{ Sample Case } & Sample Mean & $\begin{array}{c}\text { Sample } \\
\text { Standard } \\
\text { Deviation }\end{array}$ \\
\hline In Plane Resonance (Fixture) & $331.6 \Omega$ & $30.0 \Omega$ \\
\hline In Plane Resonance (Soldered) & $94.0 \Omega$ & $9.8 \Omega$ \\
\hline In Plane Anti-resonance (Fixture) & $72.4 \mathrm{mS}$ & $7.4 \mathrm{mS}$ \\
\hline In Plane Anti-resonance (Soldered) & $198 \mathrm{mS}$ & $33.3 \mathrm{mS}$ \\
\hline
\end{tabular}
the thickness resonances do not appear to follow a Gaussian distribution.

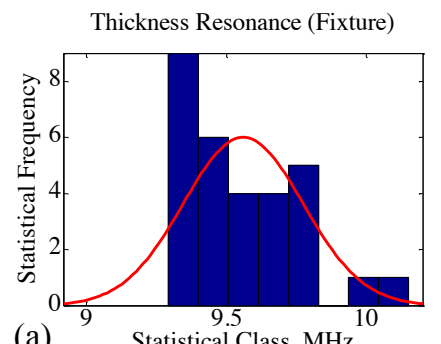

(a)

Statistical Class, $\mathrm{MHz}$

Thickness Resonance (Soldered)

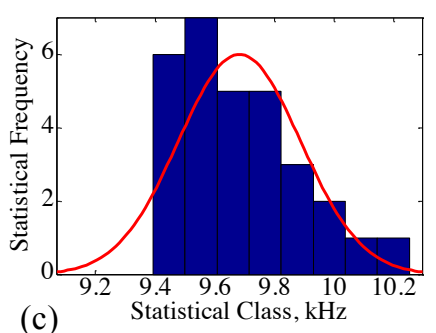

(c)
Thickness Anti-resonance (Fixture)

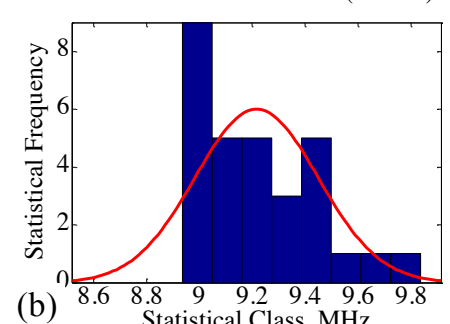

(b) $\begin{array}{llllll}8.6 & 8.8 & 9 & 9.2 & 9.4 & 9.6 \\ & & \text { Statistical Class, } & 9.6 \\ & & & \end{array}$

Thickness Anti-resonance (Soldered)

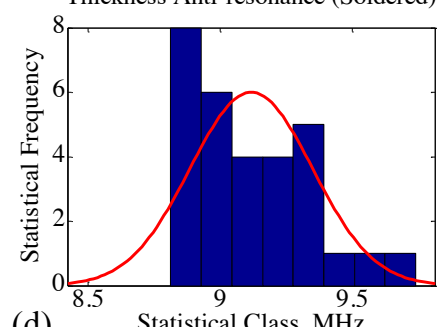

(d)

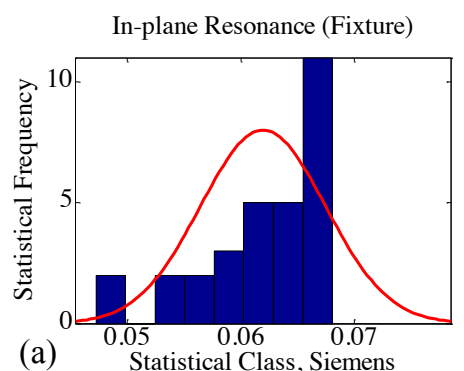

(a)

In-plane Resonance (Soldered)

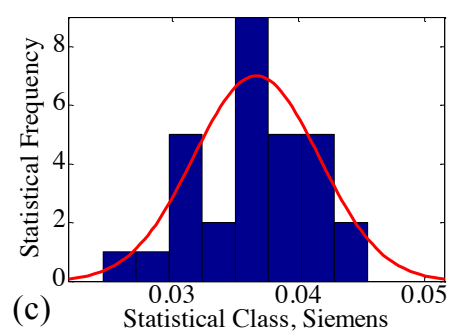

(b)

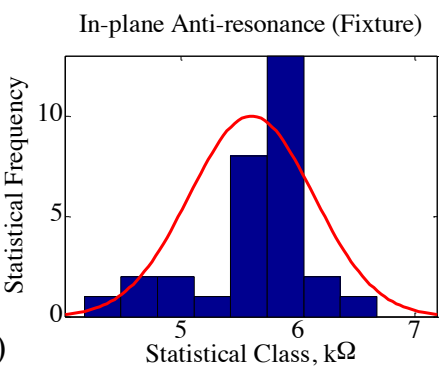

In-plane Anti-resonance (Soldered)

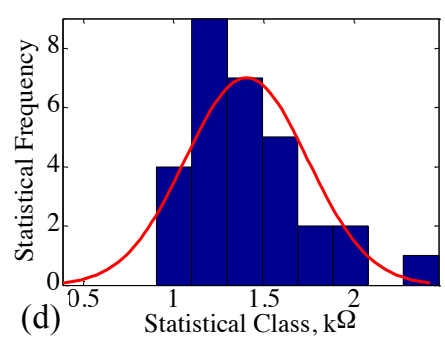

Figure 9 Histograms for in-plane resonance and anti-resonance amplitudes.

Figure 8 Histograms for thickness frequencies. 


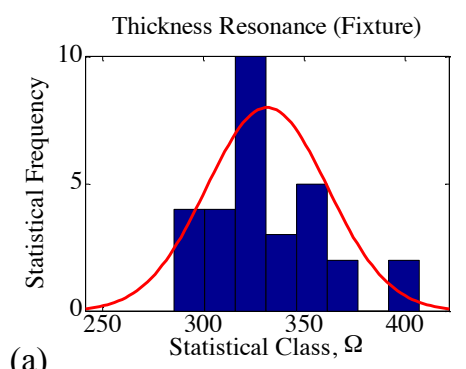

(a)

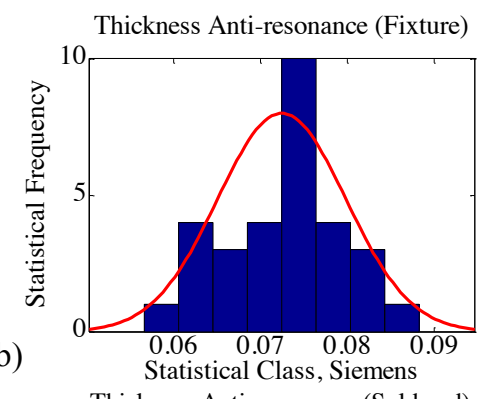

Thickness Resonance (Soldered)

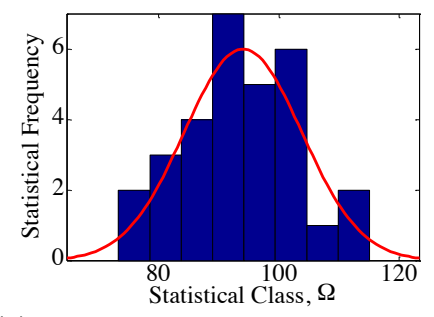

(c)

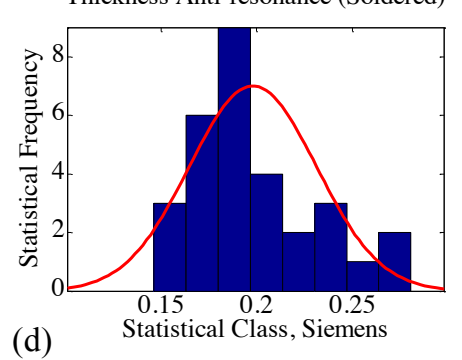

(d)

Figure 10 Histograms for thickness resonance and anti-resonance amplitudes.

\section{PWAS Analytical Model}

Presented analytical model is focused on PWAS electro-mechanical impedance as these curves govern frequency-dependent behavior of an active element. Sensors features a feedback electrode flipped over thickness of the sensors to facilitate access from one (top) side of the sensor. While sensors with feedback electrode were measured during laboratory tests, our modeling considers a circular sensor with uniform electrodes on the opposite sides of the sensor. An electro-mechanical model of such sensor can be considered for free-free sensor boundary conditions and vertical polarization direction. Solution of the equation of motion for a given set of boundary conditions yields expression for the in-plane vibration admittance [1].

$$
Y(\omega)=i \omega C\left(1-k_{p}^{2}\right)\left[1+\frac{k_{p}^{2}}{1-k_{p}^{2}} \frac{\left(1+v_{a}\right) J_{1}\left(\varphi_{a}\right)}{\varphi_{a} J_{0}\left(\varphi_{a}\right)-\left(1-v_{a}\right) J_{1}\left(\varphi_{a}\right)}\right]
$$

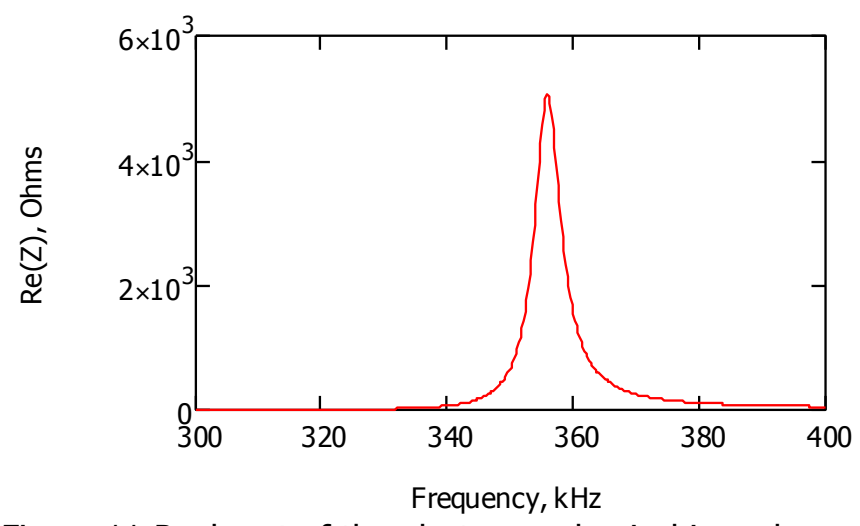

Figure 11 Real part of the electro-mechanical impedance of thin disk piezoelectric sensor. where $\phi_{a}=\omega r_{a} / c_{p}$ and $k_{p}$ is the planar coupling factor. The sound speed depend on piezoelectric density, elastic constant and Poisson ratio: $c_{p}=\sqrt{\left[\rho_{a} \cdot s_{11}^{\prime} \cdot\left(1-v^{2}\right)\right]^{-1}}$. The electrical impedance is the inverse of the admittance $Z(\omega)=[Y(\omega)]^{-1}(4)$. Substitution of typical values for APC piezoceramic result the graph depicted in Figure 11. As it could be seen from the figure, theoretical and experimental impedance are comparable in both amplitude and frequency values.

\section{MAGNETO-ELASTIC ACTIVE SENSOR AND MAGNETO-MECHANICAL IMPEDANCE TESTS}

Emergence of a new magneto-mechanical impedance (MMI) technology [3] prompted study on measurement repeatability with magneto-elastic active sensors (MEAS) [4]. MEAS consists of a coil and permanent magnet (as illustrated in Figure 12), and is used to generate elastic waves via the eddy current mechanism [5].

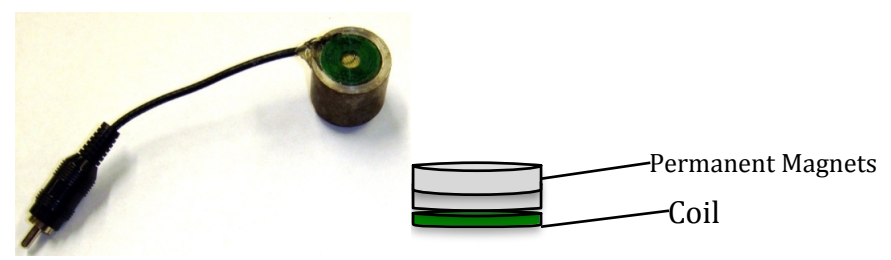

Figure 12 Magneto-Elastic Active Sensor (MEAS)

The MMI technique evaluates structural condition by considering an impedance signature that contains structural dynamic response of a metallic structure. The harmonic elastic waves transmitted by MEAS into the structure reflect off of the boundaries and produces standing (modal) spatial patterns at respective resonant frequencies [3]. The MEAS measures relevant frequency-dependent response presented in terms of dynamic impedance. It has been shown [6] that the MMI technique effectively reveals changes in the structural health signature. One notable change is a shift in the impedance frequency peaks as well as a change in the amplitude of each peak.

As a result of the transduction method, factors such as variation in sensor placement, distance between MEAS and the structure, structural geometry, etc. affect the dynamic response of the structure - notably the location of each impedance frequency peak. Therefore, this study explores how the variability in the setup affects the impedance signature. Due to the limitations in manufacturing the MEAS, one MEAS was used in order to conduct three separate experiments using fifteen trials each.

\section{Experimental Setup}

Three separate impedance tests were conducted. The equipment utilized in the experimental setup included the C7MEAS, an HP4192A Impedance Analyzer, and a computer with Labview. The MMI measurements were taken 
automatically using Labview and the acquired data were then processed in Matlab. The parameters of the C7-MEAS were as follows: diameter $-25.4 \mathrm{~mm}$, thickness $25.32 \mathrm{~mm}$, number of layers - 9, number of turns - 205.

In the first test, an aluminum 2024-T3 beam of the following dimension length $-203 \mathrm{~mm}$, width $-30.965 \mathrm{~mm}$, and thickness - $1.605 \mathrm{~mm}$, was placed with minimal contact between two foam blocks to approximate the free-free boundary conditions shown in Figure 13.

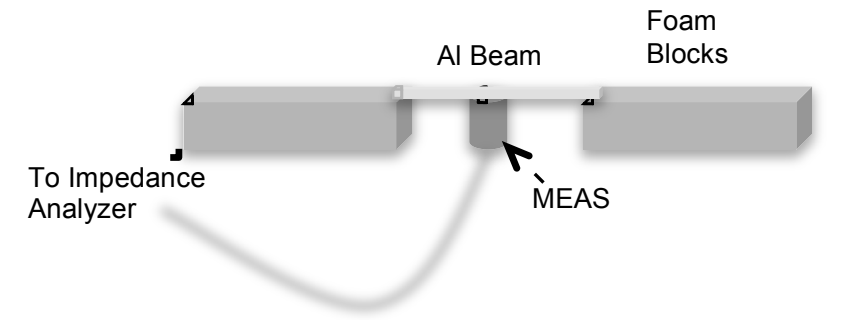

Figure 13 Experimental setup for aluminum MMI experiment.

As it can be seen in the figure, the MEAS was placed under the beam centered in the middle, approximately $0.5 \mathrm{~mm}$ under the beam. The impedance of MEAS was measured in a frequency range between $0.10-10 \mathrm{kHz}$ with a resolution of 0.10 $\mathrm{kHz}$. The beam was then removed, placed back onto the foam blocks, and impedance data were taken again. The experimental procedure was repeated for a total of fifteen tests. The acquired impedance versus frequency is presented in Figure 14.

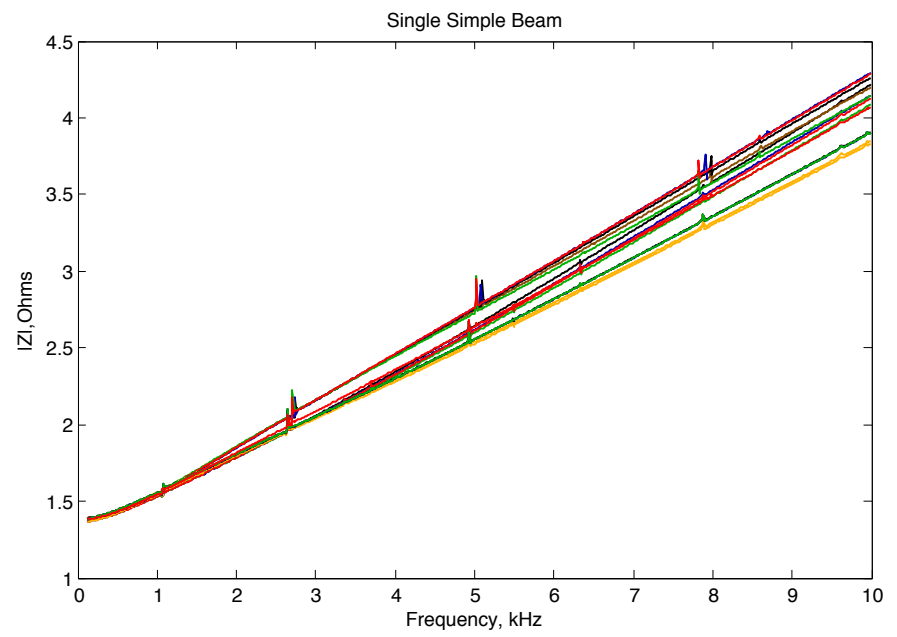

Figure 14 Impedance responses of an aluminum beam in identical experiments.

The impedance data in Figure 14 shows that the peaks deviate slightly during each test, and the slope is lower for several tests, especially the higher frequencies. The slope difference is most likely caused by a slight variation in sensor placement location, as well as distance between the MEAS and aluminum sample. In order to better understand the results, a histogram of the frequency location for each impedance frequency peak was created and presented in Figure 15.
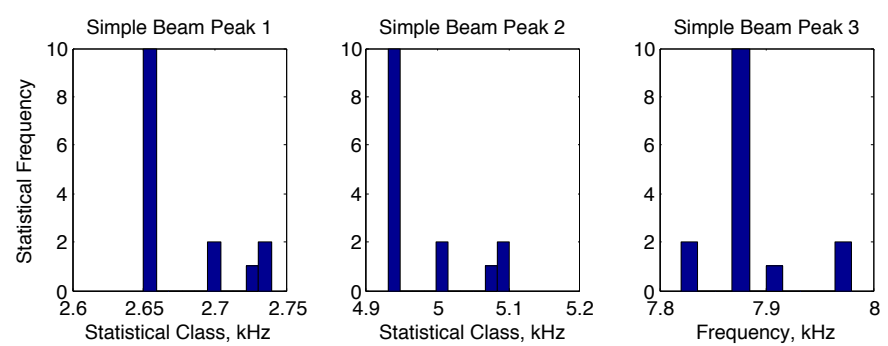

Figure 15 Histograms of impedance peak distribution for several frequencies in impedance response of the aluminum beam.

Analysis of Figure 15 suggests that majority of the impedance peaks occurred at the same frequency, with a few deviations of the order of $0.1 \mathrm{kHz}$. Since the majority of the peaks overlap, it can be concluded that there is only a slight deviation in peak location based on sensor and sample placement.

In practical diagnostics, samples of simple geometry are rare. For this reason, measurement repeatability tests were also conducted for samples of more complex geometry - dogbone specimens typically utilized in tensile fatigue test. These specimens were fabricated in accordance with ASTM-557 standard and the relevant geometry is shown in Figure 16.

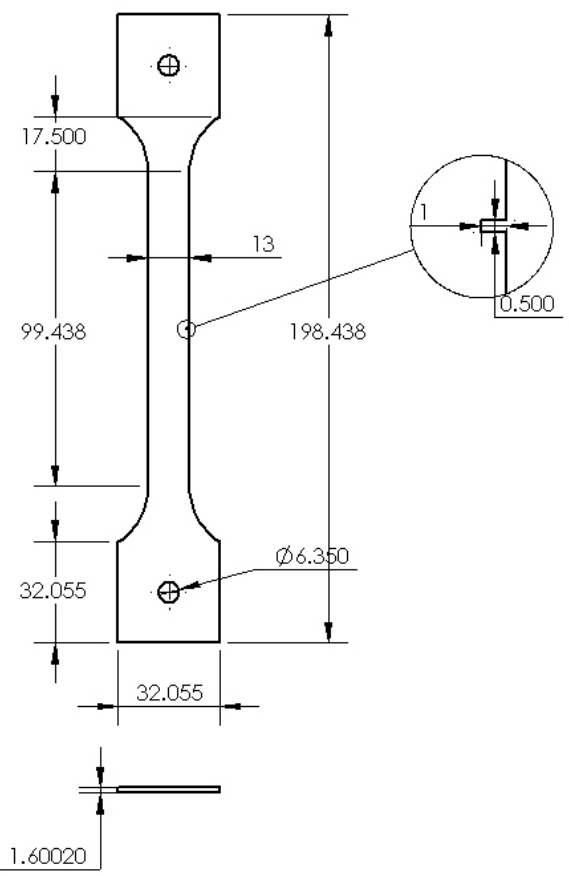

Figure 16 Geometry of dog-bone samples fabricated in accordance with ASTM-557 standard.

The MMI test with one dog-bone specimen was conducted using similar experimental settings as for the aluminum beam specimen. The sample was placed above the MEAS located between two foam blocks with minimal contact to approximate free-free boundary conditions. During the test, impedance was 
measured by sweeping frequency of the harmonic excitation in $0.1-10 \mathrm{kHz}$ frequency ranges with a frequency resolution of 0.1 $\mathrm{kHz}$. After taking the data, the sample was removed from its position on the foam blocks and placed back at the same location; impedance data were measured again. This procedure repeated for a total of 15 runs. The frequency response as a function of impedance is shown in Figure 17.

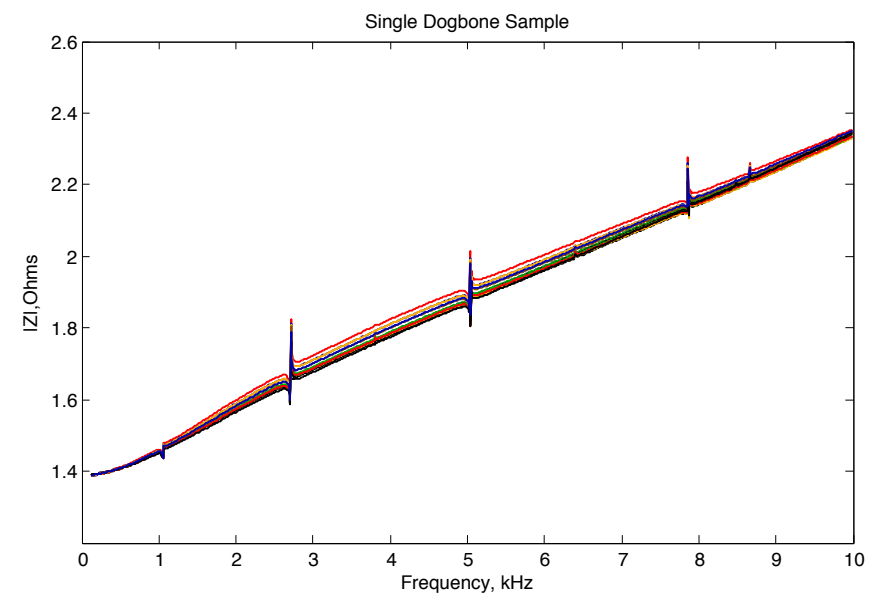

Figure 17 Fifteen MMI responses of a single dog-bone sample measured in an identical test setup.

The single dog-bone sample had almost no changes in slope, and negligible deviation in resonant frequency peaks, less than $0.1 \mathrm{kHz}$. The histogram of frequencies associated with each impedance peak location is shown in Figure 18.
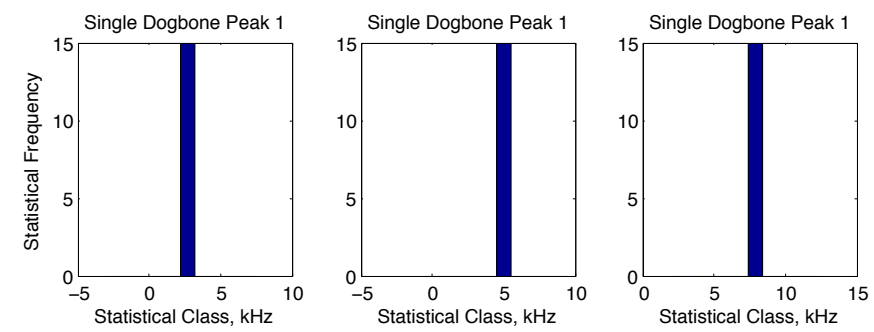

Figure 18 Histograms of impedance peak distribution for several frequencies in impedance response of the single dog-bone sample.

Noticeable in Figure 18, the resonant frequency peaks occur at the same frequency for all fifteen trials. Although in reality there may be minor variance in the peak locations, the sampling resolution was not high enough to allow for any noticeable shifts. Therefore, with this test, the repeated placement of the dog-bone sample yielded negligible changes in frequency response.

The third and final test was conducted using fifteen nominally identical dog-bone specimens, each with the geometry shown in Figure 16. After each test, a different new sample was placed between the foam blocks and tested in the previously indicated frequency band. The impedance response of the separate dog-bone samples as a function of impedance is presented in Figure 19.

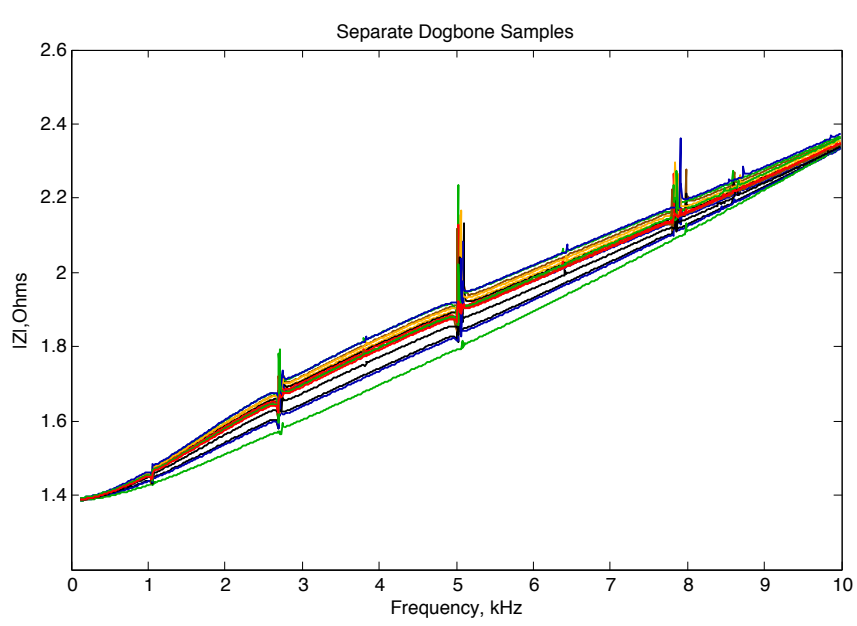

Figure 19 MMI responses of fifteen separate dog-bone sample measured in an identical test setup.

As one would anticipate, there is a slight difference in both slope and impedance frequency peaks between each sample. Both parameters are apparently affected by small discrepancies in individual sample geometries and imperfections in sample placement on foam blocks. Analysis of Figure 19 produced histograms illustrated in Figure 20.
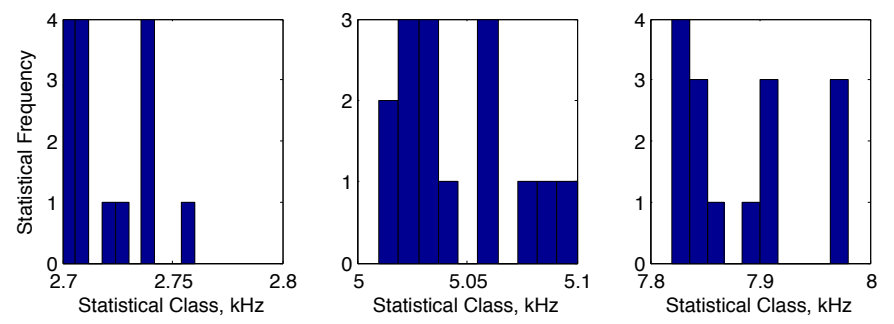

Figure 20 Histograms of impedance peak distribution for several frequencies in impedance response of fifteen dog-bone sample.

The differences between impedances of the fifteen separate dog-bone samples are evident in the slight variations in peak location between each sample. Noticeable, these differences increase at higher frequencies as shorter wavelengths are more sensitive to geometrical irregularities.

The deviation between each peak is important to know in order to differentiate between peak shifts due to structural damage, and peak shifts due to imperfection of an experimental setup. Table 4 shows a summary of the sample mean and standard deviation for each of the three tests. The maximum frequency deviation was shown to be $0.0657 \mathrm{kHz}$ for the aluminum beam sample and $0.0602 \mathrm{kHz}$ for separate dogbones. The known deviations can then be used as a baseline for expected deviation between peaks during experimentationallowing for a more definite differentiation between actual structural damage and experimental error. Current data suggest that impedance peak frequency difference between a single 
sample in repeatable tests and many nominally identical samples is negligible.

Table 4 Sample mean and standard deviation for each MMI test.

\begin{tabular}{|c|c|c|c|c|}
\hline & \multicolumn{2}{|c|}{ Peak1 } & \multicolumn{2}{|c|}{ Peak2 } \\
\hline & $\begin{array}{l}\text { Mean } \\
(\mathrm{kHz})\end{array}$ & $\begin{array}{c}\text { Standard } \\
\text { Deviation } \\
(\mathrm{kHz})\end{array}$ & $\begin{array}{l}\text { Mean } \\
(\mathrm{kHz})\end{array}$ & $\begin{array}{c}\text { Standard } \\
\text { Deviation } \\
(\mathrm{kHz})\end{array}$ \\
\hline $\begin{array}{c}\text { Aluminum } \\
\text { Beam }\end{array}$ & 2.674 & 0.0368 & 4.9720 & 0.0657 \\
\hline $\begin{array}{c}\text { Single } \\
\text { Dogbone }\end{array}$ & 2.710 & 0 & 5.0300 & 0 \\
\hline $\begin{array}{c}\text { Separate } \\
\text { Dogbones }\end{array}$ & 2.720 & 0.0194 & 5.0440 & 0.0292 \\
\hline & \multicolumn{2}{|c|}{ Peak3 } & & \\
\hline & $\begin{array}{l}\text { Mean } \\
(\mathrm{kHz})\end{array}$ & $\begin{array}{c}\text { Standard } \\
\text { Deviation } \\
(\mathrm{kHz})\end{array}$ & & \\
\hline $\begin{array}{c}\text { Aluminum } \\
\text { Beam }\end{array}$ & 7.8873 & 0.0442 & & \\
\hline $\begin{array}{c}\text { Single } \\
\text { Dogbone }\end{array}$ & 7.8600 & 0 & & \\
\hline $\begin{array}{c}\text { Separate } \\
\text { Dogbones }\end{array}$ & 7.8833 & 0.0602 & & \\
\hline
\end{tabular}

\section{CONCLUSIONS}

In this paper, piezoelectric sensor statistics and statistics relevant to repeatability of magneto-elastic impedance tests were considered.

Soldering of leads to the piezoelectric wafer active sensor significantly affects its dynamic response. Because of soldering, amplitude of impedance peaks reduces approximately 3 times, additional peaks appear in the impedance response, frequency of main resonance/antiresonance changes and impedance curves show larger deviation. In-plane resonance and anti-resonance frequencies generally conform to Gaussian distribution. However, thickness resonance and anti-resonance data does not follow the Gaussian distribution and show noticeable skewedness towards low frequencies. A simplified analytical model shows reasonable correlation with experimental data.

MMI tests conducted for 3 different setups involving a single specimen measured repeatedly and multiple specimens measured separately have shown insignificant difference for the deviation of impedance peak frequencies. In other words, there is a negligible difference between MMI responses of a single specimen and nominally identical specimens. According to the experimental data, such difference did not exceed $0.1 \mathrm{kHz}$ for the range of 0.1 to $10 \mathrm{kHz}$. Therefore, it is advocated that any changes in impedance peak frequencies that exceed this value would be likely due to structural damage rather than imperfections of the experimental setup.

\section{ACKNOWLEDGMENTS}

Authors would like to acknowledge the sponsorship of the Federal Aviation Administration (FAA) through the Center of Excellence for Commercial Space Transportation and Air Force Research Laboratory under agreement number FA945309-1-0318. The views and conclusions contained herein are those of the authors and should not be interpreted as necessarily representing the official policies or endorsements, either expressed or implied, of Air Force Research Laboratory or the U.S. Government.

\section{REFERENCES}

[1] ANSI/IEEE Standard on Piezoelectricity, 1987.

[2] Black, K., Business statistics: Contemporary decision making, Wiley, 2009.

[3] Zagrai, A. "Magneto-Mechanical Impedance of Metallic Structures," Journal of the Acoustical Society of America (JASA), Vol. 125, N. 1, January 2009, pp. EL1-EL7.

[4] Barnes, T. "Development and Application of MagnetoElastic Active Sensors for Structural Health Monitoring," MS Thesis, New Mexico Institute of Mining and Technology, 2009.

[5] Banik, N.C., Overhauser, A.W., "Electromagnetic generation of ultrasound in metals", Physical Review B, 16 (8) (1977) 3379-3388.

[6] Zagrai, A. and Çakan, H., "Magneto-mechanical Impedance Identification and Diagnosis of Metallic Structures," International Journal of Engineering Science, Vol. 48, Issue 10, October 2010, pp. 888-908. 